\title{
Broad Spectrum Chemistry as Practised by Novartis Process Research
}

\author{
Stuart J. Mickel ${ }^{\star}$, Reto Fischer, and Wolfgang Marterer
}

\begin{abstract}
Three actual examples from the current product palette within Novartis Process Research will demonstrate the some of the variety and challenges encountered in modern chemical development.
\end{abstract}

Keywords: Aldol $\cdot$ Dakin-West $\cdot$ Dimroth $\cdot$ Discodermolide $\cdot$ Nitration

\section{Introduction}

Modern chemical development faces a formidable challenge in converting the wonderful set of reactions and reagents which are published in the chemical literature every day, into workable reliable processes. Normally this 'state of the art' chemistry is described to function, usually on a very small scale, but its conversion to a scaleable reproducible process provides the development chemist with a set of severe problems. The variety of structures that can be encountered by the development chemist is vast and ranges from antibiotics to tertasaccharides. The array of issues with which a development chemist is confronted is equally as large, from environmental/health and safety concerns to polymorphism and the properties of solids. In this article we would like to highlight some of these challenges by referring to a selection of three recent projects from our department. These will demonstrate how some of the issues encountered in the daily business of chemical development may be solved.

In the first example a short overview of the development history of a relatively simple compound will show how the elaboration of alternative synthesis concepts - even in the field of well-known heterocycles can indeed lead to economically as well as ecologically superior manufacturing processes. It also demonstrates the value of re-examining 'old' reactions and shows how mechanistic considerations can be of immense value in attaining a safe reproducible scalable process.

\section{CGP59326 New Process}

Pyrrolo[2,3-d]pyrimidine-derivative $\mathbf{1}$ (Fig.1) was manufactured in $100 \mathrm{~kg}$ scale for clinical investigation as an exploratory anti-tumour agent [1].

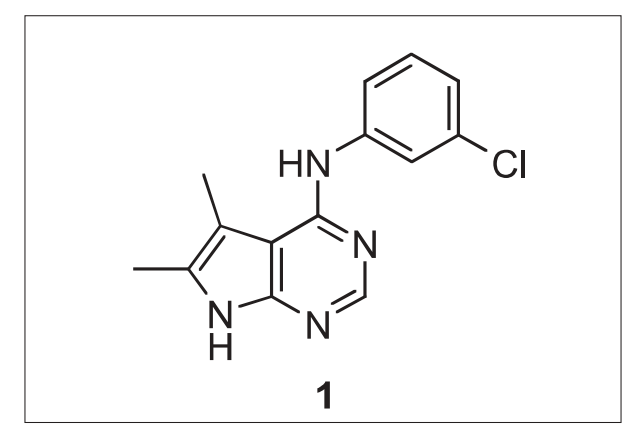

Fig. 1.
The synthesis from research (Scheme 1) was relatively straightforward, however, for the following main reasons, we elaborated an improved, alternative synthesis for scale-up in the pilot plant and towards future production:

1) The use of a benzyl protecting group for the pyrrole amine functionality was mandatory for the outlined synthetic strategy, however, deprotection turned out to be technically unfavourable because it required a large excess of aluminium trichloride which subsequently had to be quenched and disposed of.

2) The classical, three-component pyrroleforming condensation reaction was lowyielding.

3) The pyrimidine cyclisation step in boiling formic acid raised safety concerns and resulted in dark coloration of the product which made cumbersome purification procedures necessary.

4) The hydroxy- as well as the chloropyrimidine intermediates had extremely poor solubility which resulted in dilute

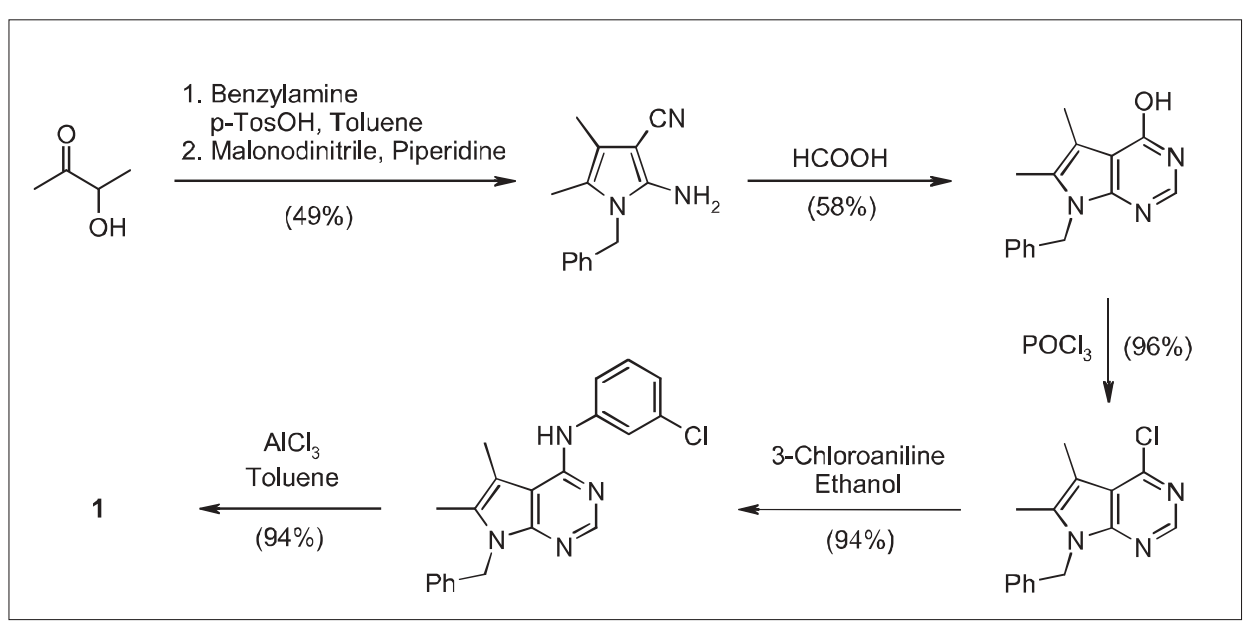

Scheme 1.

\footnotetext{
${ }^{\star}$ Correspondence: Dr. S.J. Mickel

Chemical and Analytical Development

Novartis Pharma AG

$\mathrm{CH}-4002$ Basel

Tel.: + 41616962952

Fax: + 41616962957

E-Mail: stuart_john.mickel@pharma.novartis.com
} 
reactions and a large excess of phosphorus oxychloride being used for the chlorination step.

\subsection{Development Synthesis Strategy}

For the synthesis of $\mathbf{1}$, we concluded that 2-amino-3-cyano-4,5-dimethylpyrrole (2) (Fig. 2) was the intermediate of choice. Its simple synthesis from 3-amino-2-butanone and malonodinitrile was already described more than 40 years ago [2] and it is sufficiently stable towards air oxidation to allow normal handling in a production facility.

From this pyrrole we intended to modify the pyrimidine ring formation in such a way that no protection was necessary and the need for formic acid was eliminated.

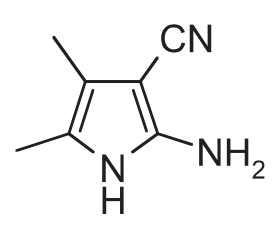

2

Fig. 2.

\subsection{Pyrrole Synthesis}

$\alpha$-Acylamino ketone 3 [3] is not commercially available but easily accessible from alanine and acetic anhydride, a reaction that was initially described by Dakin and West in 1928 [4] and therefore is known as the Dakin-West reaction. Since (racemic) alanine is very cheap, this seemed to be an attractive approach to the synthesis of pyrrole $\mathbf{2}$ (Scheme 2).

However, there is one major drawback to the Dakin-West reaction regarding its scale-up to plant scale: it is accompanied by an uncontrolled formation of carbon dioxide, which is absolutely prohibitive on technical scale. Our initial attempts to control formation of carbon dioxide by variation of reaction parameters all failed and additionally never produced yields which were at least comparable to the standard protocol. So we decided to examine the reaction mechanism in more detail.

Even though the Dakin-West reaction can hardly be considered well-known, there is quite a lot of published work including some very detailed mechanistic investigations particularly by Steglich [5]. It is generally accepted that the mechanism involves dehydrative formation of an azlactone (oxazolinone) which is then $\mathrm{C}$ acylated (in equilibrium with O-acylation), and then undergoes ring-opening hydrolysis followed by decarboxylation to form the acylamino ketone (Scheme 3) [6].

After some of our own investigations confirmed the mechanism outlined above, we concluded that the following kinetic interpretation would at least partly explain the peculiarities of the Dakin-West reaction: as we see in Scheme 3, the ring-opening hydrolysis of acylated azlactone formally requires 1 equiv. of water. Since there are certainly not sufficient quantities of water in a boiling reaction mixture of excess anhydride and pyridine, this 'water-equivalent' has to come from the formation of acetic anhydride from acetic acid. Acetic acid itself is formed in the first two steps of the reaction sequence, namely $\mathrm{N}$-acylation and azlactone condensation. Therefore its initial concentration in the reaction mixture is very low and hence azlactone hydrolysis is very slow. Once the mixture is heated and $\mathrm{N}$-acylation of alanine starts to increase the acetic acid concentration increases rapidly and therefore azlactone hydrolysis is accelerated. The following decarboxylation is virtually spontaneous. This results in the reaction having a typical, self-accelerating kinetic, which is exactly what you do not want to have in a pilot or production plant, particularly not if equivalent amounts of gas are formed during a reaction.

To test this hypothesis, we performed experiments where we added various amounts of acetic acid to the initial mixture of acetic anhydride and pyridine - an idea which surprisingly has never been investigated in the

literature. The results were convincing: 1 equiv. of acetic acid made it possible to run the reaction in an addition-controlled mode with carbon dioxide evolution being directly linked to alanine addition. As an extra benefit, acetic anhydride and pyridine excess could now be significantly reduced.

In a second step, the reaction was successfully adapted to the modified DakinWest procedure with triethylamine/DMAP, introduced by Steglich and Höfle in 1969 [7] which requires much smaller amounts of anhydride and base and is therefore economically more advantageous. Eventually, over $0.5 \mathrm{kmol}$ alanine were successfully converted to acetylaminobutanone $\mathbf{3}$ in a 4001 reactor in over $90 \%$ yield with carbon dioxide evolution ( 11'000 1!) being in a virtually linear relation to alanine addition. The crude product solution was concentrated by evaporation of acetic acid, acetic anhydride, and triethylamine. This concentrate was diluted with water and malonodinitrile and the resulting solution added to $30 \%$ sodium hydroxide whereby pyrrole 2 was formed and precipitated in an extremely fast, exothermic but fully addition-controlled reaction [8]. Crystalline pyrrole 2 was directly isolated by centrifugation, washing with water and drying and did not require further purification [9]. Neither distillate nor mother liquor required any special treatment. This procedure for the synthesis of pyrrole $\mathbf{2}$ (Scheme 2 ) is very efficient, high-yielding (83-85\% over 2 steps calculated from alanine) and requires only two standard reactors.

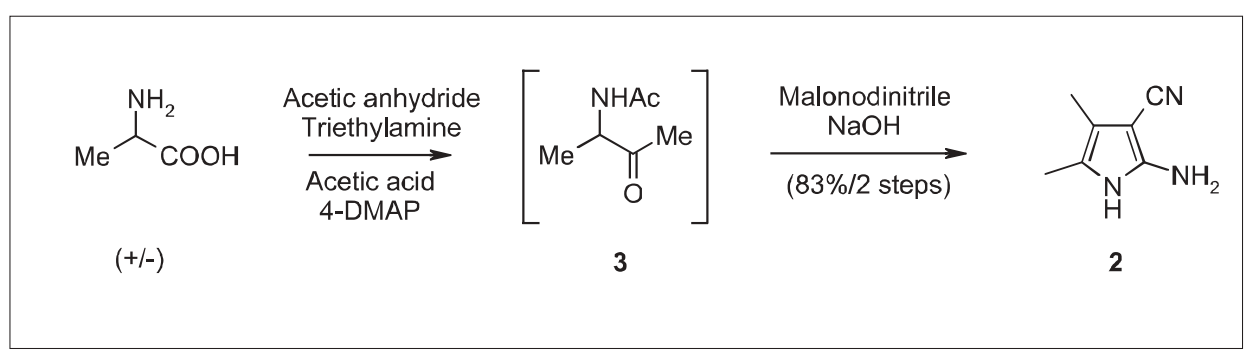

Scheme 2. Pyrrole synthesis

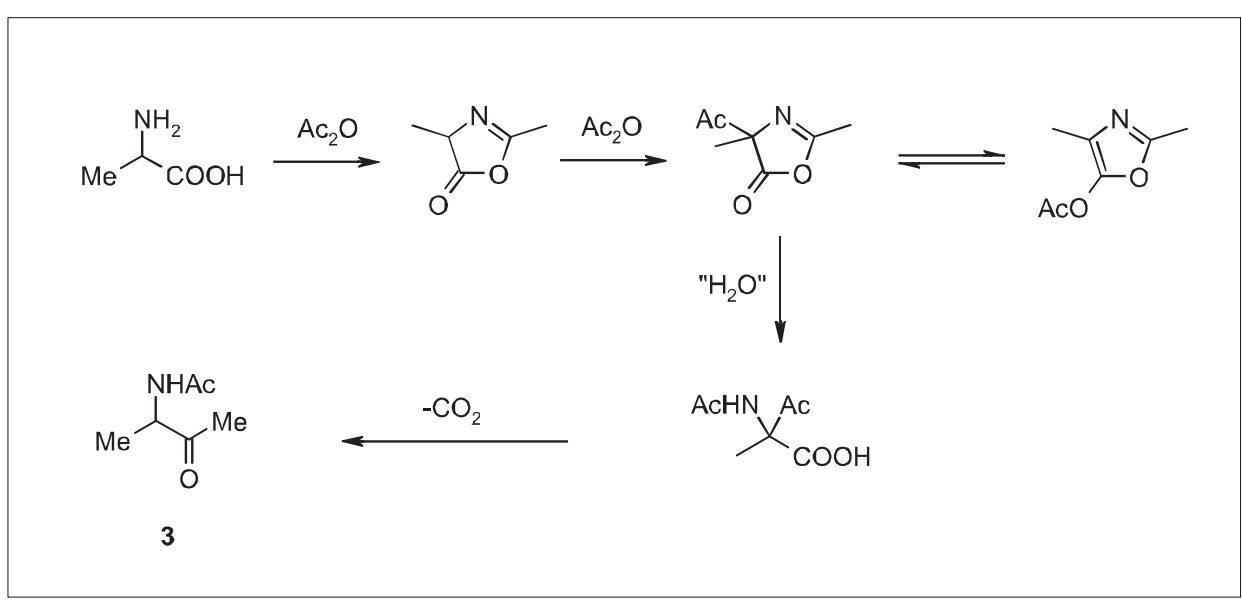

Scheme 3. Published mechanism of the Dakin-West reaction 


\subsection{Pyrimidine Ring Synthesis}

In 1964, Taylor and Hendess reported a procedure to convert an unprotected aminocyano-pyrrole into an aminopyrrolopyrimidine derivative with trimethyl orthoformate and ammonia [10]; however the very scarce use of this method in the newer literature seemed to indicate a somewhat limited scope. We reasoned that in principle, it should be possible to apply this type of pyrimidine ring formation to pyrrole 3 under carefully optimised conditions which avoid side reactions at the pyrrole ring nitrogen. To explore this, we initially prepared iminoester 4a (Fig. 3) from pyrrole 3 and triethyl orthoformate.

The crude iminoester was then treated with 3-chloroaniline. This sequence under various conditions at best produced moderate yields of 3-chlorophenyl-pyrrolopyrimidine 5 (Scheme 4) in rather unsatisfying quality. Therefore, the reaction sequence was changed: iminoester $\mathbf{4 b}$ was formed from 3-chloroaniline and triethyl orthoformate under acid catalysis [11] and isolated as a crude oil. Pyrrole $\mathbf{3}$ was then treated with iminoester $\mathbf{4 b}$. This procedure turned out to be superior particularly after optimisation of solvent, acid catalyst and $\mathrm{pH}$.

We concluded that the mechanism of this pyrimidine ring formation involves a series of orthoester-iminoester-amidine equilibria. Hence, it should even be possible to run the reaction under kinetic control without preformation of any iminoester intermediate. In agreement with our expectations, we found, that under carefully optimised conditions, the three-component reaction of pyrrole $\mathbf{3}$, triethyl orthoformate, and chloroaniline proceeded relatively slowly but was surprisingly clean. This formation of pyrrolopyrimidine 5 turned out to be distinct from the initial procedure because only trace amounts of iminoester $\mathbf{4 b}$ were detected in the reaction mixture. Instead, a new, major intermediate was found and upon isolation identified as amidine $\mathbf{4 c}$ which now seemed to be the reactive species towards the formation of the pyrimidine ring [12]. This optimised and simplified process was run in the pilot plant on $0.45 \mathrm{kmol}$ scale. Chloroaniline and triethyl orthoformate were dissolved in ethanol and the $\mathrm{pH}$ of the solution was adjusted to 5.5 by addition of acetic acid. Pyrrole $\mathbf{3}$ was then added over a few hours at $45-50{ }^{\circ} \mathrm{C}$; after cooling, precipitated pyrrolopyrimidine 5 was centrifuged off, washed and dried. The yield in the pilot plant was over $80 \%$ (based on pyrrole $\mathbf{3}$ ).

Chlorophenyl-pyrrolopyrimidine 5 now only needed to be converted to its isomer 1 .

This isomerisation is referred to as the Dimroth rearrangement [13]. For pyrrolopyrimidines, it is very rarely reported in the literature [14], with only a few more examples for other pyrimidine derivatives [15]. Lab trials led to the conclusion that in this particular case, no catalyst was required and reaction temperature was the most important parameter. A mixture of ethylene glycol, ethanol, and water eventually turned out to be optimal; clean isomerisation could be achieved in over $90 \%$ yield by simply refluxing for a few hours. Upon cooling, the product precipitates and can once again be isolated as a white solid by filtration, washing and drying. This procedure was successfully run in the pilot plant in $0.3 \mathrm{kmol}$ scale (Scheme 4).

The drug substance free base was brought in its mesylate salt form by crystallisation from ethanol/water in the presence of methylsulfonic acid and final recrystallisation from acetone/water, yielding a white powder with high purity (HPLC assay $>99 \%$ ) which met all internal specifications and was subsequently released for use in humans.

In summary, a very efficient, cost effective and ecological process for the manufacturing of $\mathbf{1}$ was developed, which is suit- able for production in standard equipment of a multi-purpose plant [16]. In addition to the best of our knowledge - this is the first application of a Dakin-West reaction on a technical scale.

\section{Discodermolide}

The second example demonstrates the power of modern organic chemistry and also highlights some of its limitations. We were asked to prepare a significant amount of a rare natural product, (+)-discodermolide 6 (Fig. 4).

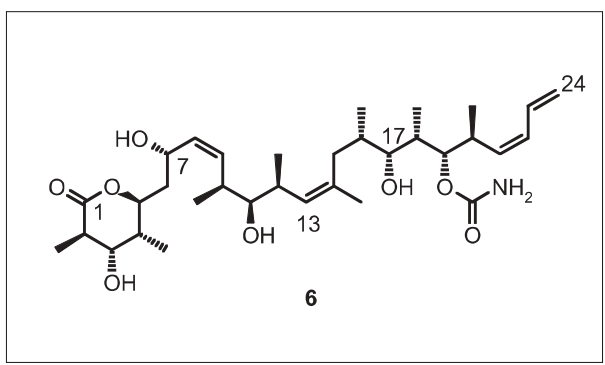

Fig. 4. (+)-Discodermolide ((+)-6)

This compound was first isolated in 1990 from a rare deep sea sponge (Discodermia dissoluta) by workers at the Harbor Branch Oceanographic Institute in Florida [17]. It is a microtubule stabilizing agent and has demonstrated efficacy in human tumour growth inhibition in hollow fibre and xenograft mouse models [18]. The compound is produced by the sponge in very small quantities around $2 \mathrm{mg} / \mathrm{kg}$ per dry weight of sponge: therefore the only reasonable way to procure quantities of this compound for clinical evaluation is total synthesis.

\subsection{Problem Analysis}

Structurally, discodermolide (6) consists of a linear polypropionate chain con-

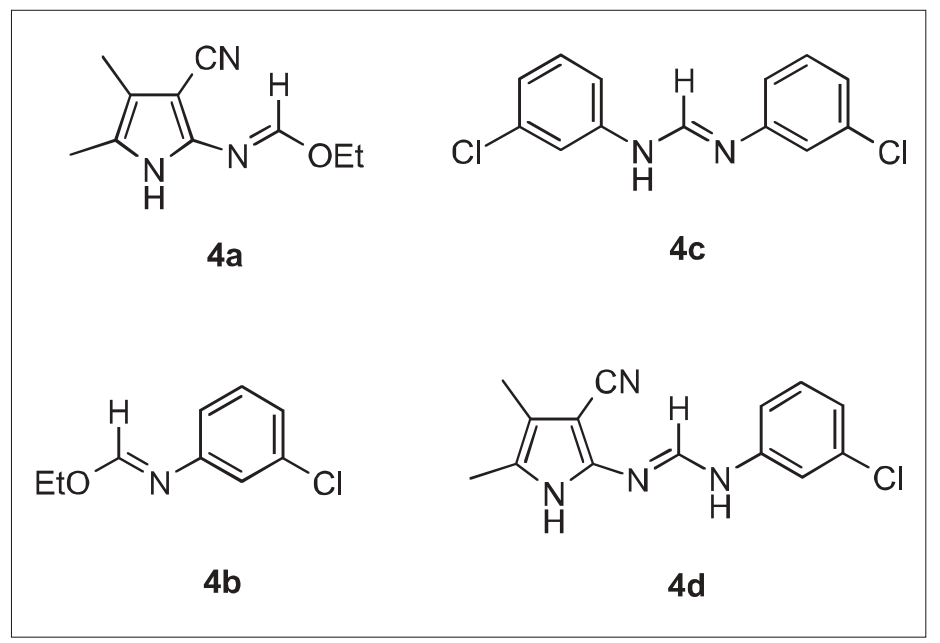

Fig. 3.



Scheme 4. Final pilot plant route 
taining thirteen stereocentres, six of which are hydroxyl-bearing, with one of these esterified as a $\gamma$-lactone $(\mathrm{C}(5))$ with another as a carbamate $(C(19))$. It also features seven methyl-bearing stereocentres and three $(Z)$ configured alkenes, one of these being part of the terminal diene unit another being a cis trisubstituted system at $\mathrm{C}(13)-\mathrm{C}(14)$ which offers a synthetic challenge in controlling the stereochemistry. Also present in the structure is a common stereo triad (methyl, hydroxyl, and methyl) that is repeated three times.

The synthesis of this compound posed a real challenge for chemical development. Alone the number of steps required (36), together with the number of chromatographic purifications (around 17) appeared to make the project prohibitive. However, in view of the projected dose we decided to go ahead with the synthesis of around $60 \mathrm{~g}$ target quantity.

We scanned the available literature and at that time discovered around five total syntheses described [19] together with a number of publications describing fragment synthesis [20]. A useful review of the available synthetic approaches has recently been published [21]. A working group was established to evaluate the chemistry described in these published approaches. We analysed them with respect to a number of criteria, e.g. problematic reagents, changes in oxidation state, number of chromatography passes and so on. Many of the reactions described were deemed to be not practicable on any sort of significant scale. For example, Smith et al. reported [19e] a highpressure reaction for the synthesis of an advanced intermediate phosphonium salt (12 Kbar), investigations on this crucial step have recently been published [22]. This was not an option for us. In some of the other routes we came across non-stereoselective reactions, low molecular weight acetylenes (thermal stability problems), lots of chromatography, and no (or very few) crystalline intermediates. One synthetic challenge was certainly the formation of the C(13)-C(14) trisubstituted cis double bond. Numerous ingenious ways have been devised to construct this structural feature. For example Paterson et al. employed a selenium Claisen rearrangement [19h], Arefolov and Panek [20b] utilised some acetylenes combined with attractive zirconium chemistry to set up this structural feature. Unfortunately these two attractive routes were not an option for us on the large scale. The crystallisation of discodermolide was also not defined by any of the authors. This is a very important point for the development chemist, as it must be ensured that the correct crystal modification is formed.

We finally settled on a combination approach of the synthesis of Prof. Smith for the first part of the route merging with that of Prof. Paterson for the latter part of the

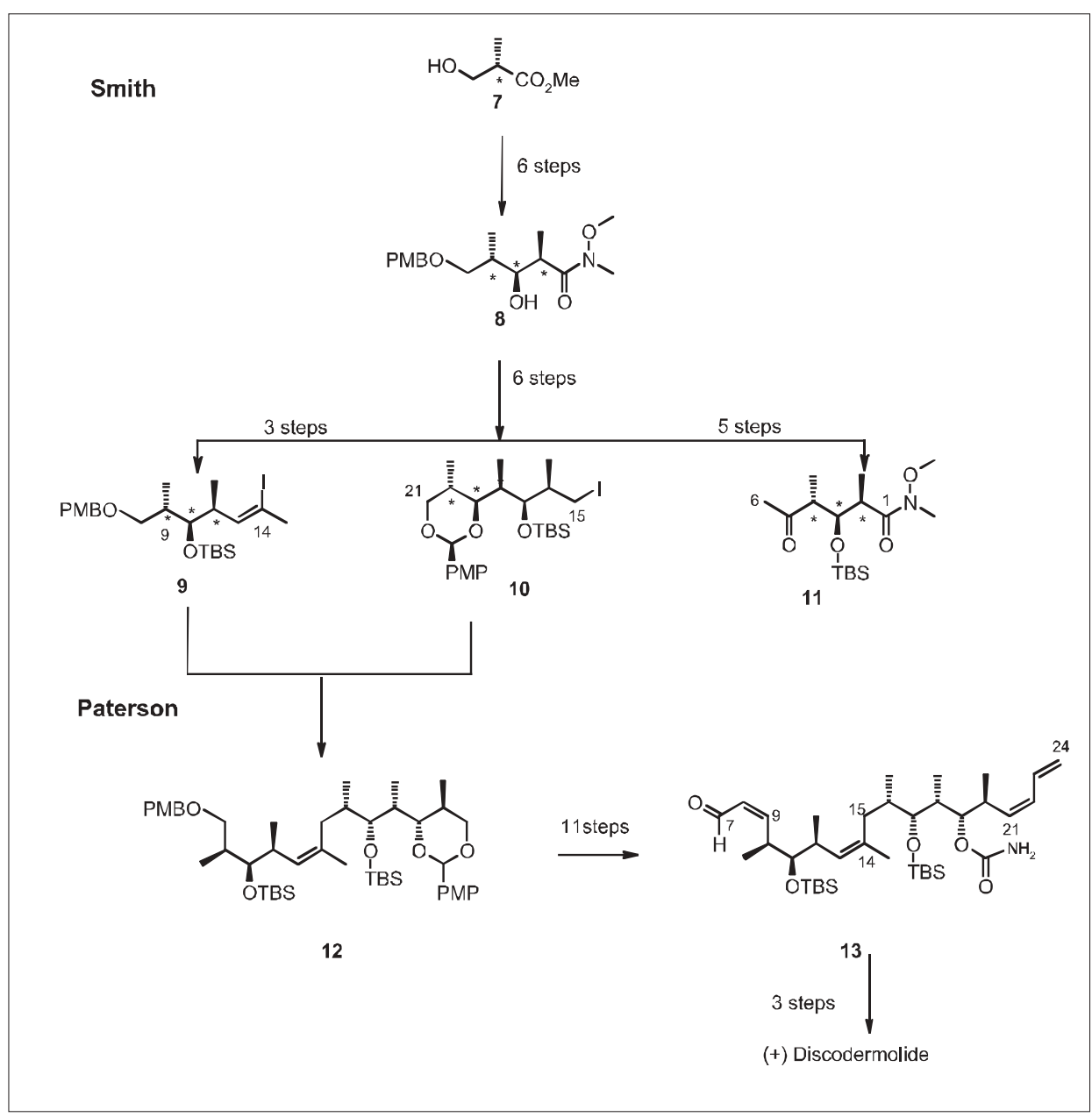

Scheme 5: Abbreviated synthetic scheme for (+)-discodermolide with numbering scheme

large-scale synthesis [23]. An abbreviated synthetic Scheme is shown in Scheme 5.

Starting from the readily available ester $\mathbf{7}$ a common intermediate, amide $\mathbf{8}$, was prepared in six steps. This compound contains the repeating stereo triad present in $\mathbf{6}$. Thus, suitable manipulation leads to the three advanced intermediates $\mathbf{9 , 1 0}$, and $\mathbf{1 1}$. After Suzuki coupling of $\mathbf{9}$ and $\mathbf{1 0}$ to produce fragment $\mathbf{1 2}$ further elaboration into the Paterson synthesis eventually produces the advanced aldehyde 13, which is then coupled in a final aldol reaction with ketone 11. Three final steps then deliver the final product 6 [23].

\subsection{Some Problem Steps}

Some of the "named bond forming reactions' used in the synthesis are shown in Fig. 5.

In this entire synthesis of some 36 steps there are many 'problem steps' which can be identified. Three of these will be briefly discussed in the following section.

\subsubsection{Cis Vinyl lodide}

As indicated above the introduction of the $\mathrm{C}(13)-\mathrm{C}(14)$ cis substituted double bond unit is especially challenging. We chose to employ the Zhao olefination [24] procedure, as described by Smith et al. [19e] and Mar-




shall and co-workers [19g], depicted in Scheme 6 to prepare the cis vinyl iodide precursor for this structural unit.

This reaction is fraught with problems, all the parameters one can imagine, e.g. time, temperature concentration, order of addition, work-up, chromatography, and so on, are critical in this process. We obtained the desired cis-vinyl iodide 9 in $31 \%$ yield after chromatography purification on silica gel. Only small amounts of the undesired trans isomer were detected (cis/trans 15:1), this can be easily removed at the next step. We did not observe any des-iodo olefin (compound A, Scheme 6) suggesting that the iodination of the phosphonium salt via ylide iodination had been completed before it was added to aldehyde $\mathbf{1 4}$.

This olefination step was one of the most difficult reactions for scale-up. We consistently obtained $25-31 \%$ yield on the maximum scale of $2.5 \mathrm{~kg}$ of aldehyde $\mathbf{1 4}$. Complicated work-up procedures and instability of 9 contributed to the low yield. Elemental iodine has been utilised for the conversion of ethyltriphenylphosphonium iodide into the iodo ylide. We found that Niodosuccinimide can be used to replace iodine without detriment. While this makes the reaction easier to handle, it did not contribute to an increase in yield. Initially, we observed the formation of the methyl ketone B [24] during the work-up. This byproduct can be avoided by using non-aqueous work-up.

Smith and coworkers [25] reported that the reaction of the iodo ylide with $\mathbf{1 4}$ afforded epoxide $\mathrm{C}$ in addition to the desired 9 in a 1:1 ratio. Alternative approaches were investigated to minimize this major byproduct, however, they were unsuccessful. For example employing a method described by Shenand Gao [26] where the initially formed betaine intermediate was deprotonated with a second equiv. of base and then iodinated, produced des-iodo olefin A. Utilizing Hanessian's phosphonates [27] in this process also resulted in only des-iodo olefin A.

\subsubsection{Paterson Aldol}

This is a mis-matched aldol reaction where the stereochemical bias of the reaction is overturned by the chiral nature of the reagent (Scheme 7) [19h][28].

This turned out to be a very capricious reaction and achieving reproducibility was (and still is) a tremendous challenge. After extensive small-scale experimentation several factors were determined to be responsible for this lack of reproducibility:

1) Reagent quality: $\mathrm{DIP}-\mathrm{Cl}$ is a low melting, extremely air and moisture sensitive unstable compound. At temperatures above $0{ }^{\circ} \mathrm{C}$ it eliminates one molecule of pinene. Depending on the reagent quality the aldol either worked

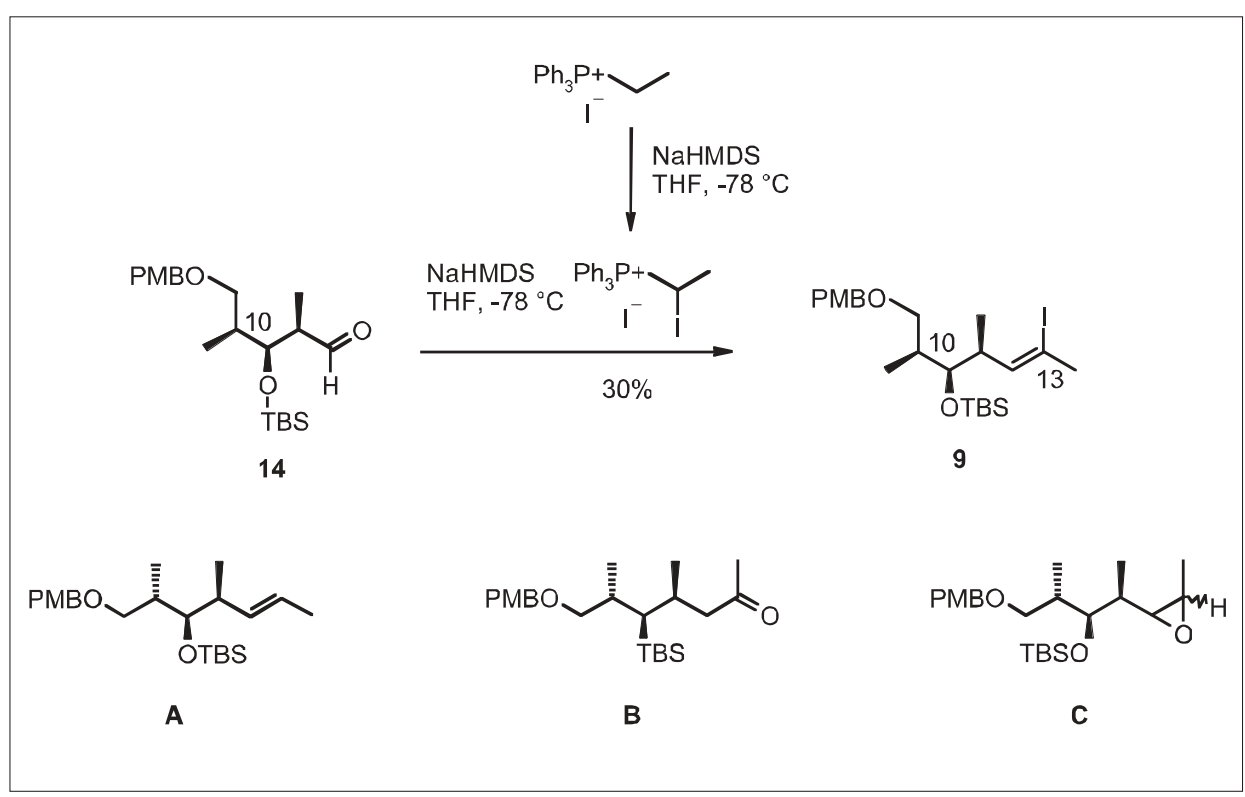

Scheme 6. Route to cis vinyl iodide

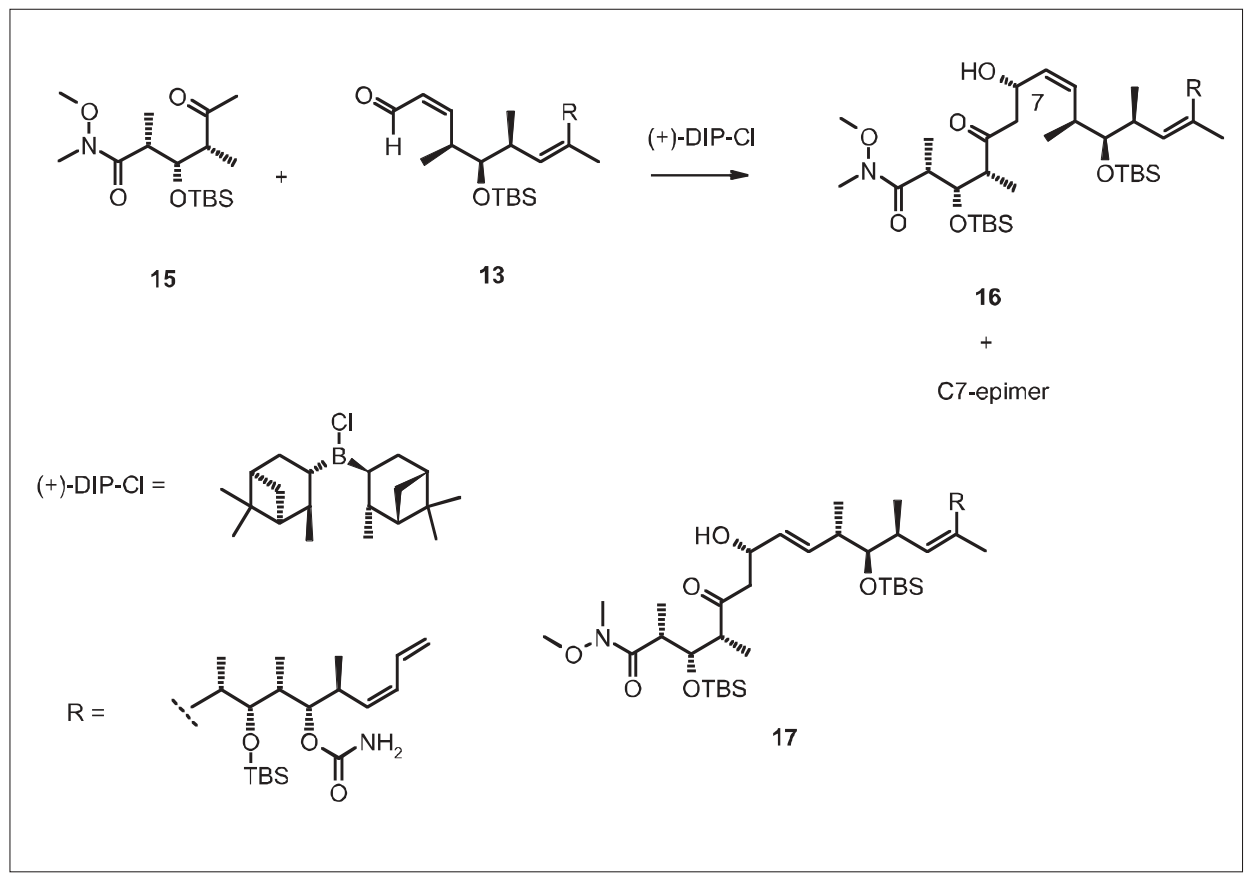

Scheme 7. Paterson aldol reaction

in low yield or not at all. The reagent quality is not definable.

2) The presence of traces of moisture causes isomerisation of cis enal nearly quantitatively to the trans enal, which then takes part in the aldol reaction producing aldol 17.

3) The enolate formation is complicated by an internal (Claisen) cyclisation reducing its availability for aldol reaction.

4) Large excesses of enolate (6.6 equiv. based on aldehyde 13) are required to push the reaction to completion, complicating work-up and purification.

5) The ratio of epimers, 3.5-4.0:1, is best obtained using diethyl ether as a solvent.
6) A solvent change is necessary before beginning the oxidative work-up.

7) Aqueous quench and evaporation of the reaction mixture (before oxidative work-up) leads to significant decomposition of the product and its epimer.

8) Oxidative work-up leads to significant decomposition of the product and its epimer.

9) The product is not very stable to chromatography on normal-phase silicagel but stable to reverse-phase conditions.

10) The product is not crystalline.

With all these problems how was a reproducible process obtained? 
A (+)-DIP-Cl supplier was found who produced the compound as a $70 \%$ solution in hexanes. This solution is, according to the manufacturer, indefinitely stabile, even at room temperature. Utilising the reagent in this form immediately solved the reproducibility problems. The reaction went to completion (complete consumption of aldehyde 13) with little to no isomerisation to the trans enal being observed! However, on scale-up (100 $\mathrm{mg}$ to $50 \mathrm{~g}$ of 13 ) the lab yield of $45 \%$ of $\mathbf{1 6}$ was not achieved, $20 \%$ being obtained.

We examined the fate of $\mathbf{1 6}$ throughout each stage of the work-up and discovered that it was apparently not stable. The reason for this instability is not clear but may be related to the large excesses of reagents which are required to drive the reaction to completion.

The solution was to eliminate the workup steps entirely and directly chromatograph the reaction mixture without workup! This provided a $60 \%$ yield of the desired epimer. Thus $700 \mathrm{~g}$ reaction mixture, containing around $12 \%$ of the desired product, was diluted with $368 \mathrm{~kg}$ of $\mathrm{MeCN} / \mathrm{TBME} / \mathrm{H}_{2} \mathrm{O}$ 85:15:10 and applied to a $120 \times 30 \mathrm{~cm}$ column containing 20 kg RP-18 reverse-phase silica-gel. The column was eluted with $1060 \mathrm{~kg}$ $\mathrm{MeCN} / \mathrm{TBME} / \mathrm{H}_{2} \mathrm{O} \quad 85: 15: 10$ then MeCN/TBME 1:1 collecting $20 \mathrm{~kg}$ fractions. The product fractions were combined and partially evaporated and the residue extracted with ethyl acetate and re-evaporated to give $150 \mathrm{~g}$ of pure aldol product $\mathbf{1 6}$. These conditions remove the 'reactive components' which presumably initiate the decomposition. Isolated $\mathbf{1 6}$ was found to be a stable compound.

\subsubsection{Final Step}

The final step in the sequence leading to (+)-6 is shown in Scheme 8.

This desilylation reaction of $\mathbf{1 8}$ (obtained by Evans-Saksena reduction [29] of 16 with tetramethylammonium triacetoxyborohydride) is very complex. The first, rapid reaction is cyclisation to $\mathbf{1 9}$ followed by slow cleavage of the various silyl groups. All the possible combinations of bis-silyl intermediates can be observed in the LC of this reaction. The slowest silyl group to hydrolyse is that at the 3-position. The reaction is a fine balance between desired and undesired processes (side product formation) and must be closely monitored.

Chromatography on reverse-phase silica-gel with an acetonitrile/water mixture delivered (+)-6 in 70\% yield. The compound thus isolated was shown by HPLC to be a mixture of lactone and the corresponding hydroxy acid (92:8). This equilibrium was readjusted completely to the lactone side by lowering the $\mathrm{pH}$ with hydrochloric acid. Thus on crystallization from acetonitrile/wa-

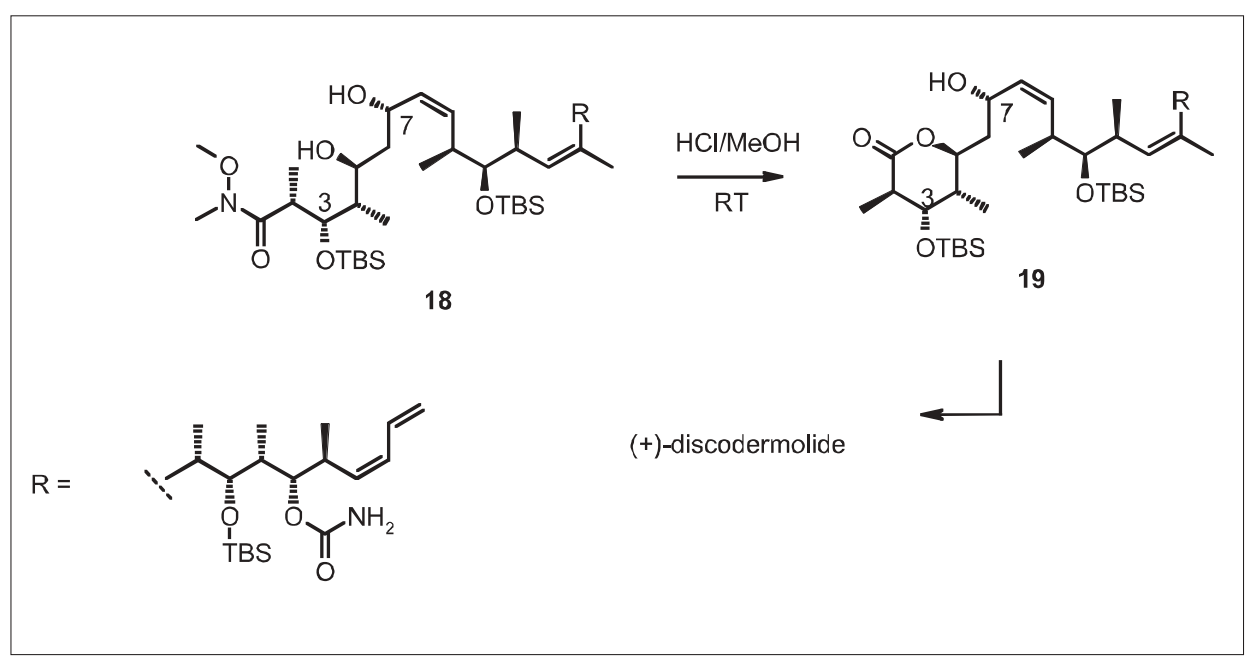

Scheme 8. Final steps of the synthesis of $(+)-6$

ter $85: 15$ at $\mathrm{pH} 4$ the lactone was the only product isolated as sandy crystals in $95 \%$ yield. The polymorphic form (mono-hydrate) that was obtained by the above recrystallisation method was highly reproducible.

Several side products are formed during the cleavage process [30] (Fig. 6), they are easily separated by chromatography. Treatment of (+)-6 itself with acid also produces the same set of compounds. Their formation demonstrates the propensity of (+)-6 to cyclise via participation of the $\mathrm{C}(13)-\mathrm{C}(14)$ double bond.

That over $60 \mathrm{~g}$ of (+)-6 could be prepared by this route is a tribute to the power of modern chemistry and its proponents. The entire process took some 20 months to complete, an average of one step per fortnight. Surprisingly, most of the steps were transferred to larger scale without any great problems. We identified around seven problematic steps, of which three occur early in the route. The end game is far from ideal, after such a synthetic sequence the final few steps leading to the final drug substance need to be kept 'simple'. The arduous chromatography of the aldol coupling product is clearly not practical to move into production, fortunately, the predicted low dosage levels should keep the yearly manufacturing requirements to a minimum.

One major problem associated with a synthesis of this length is the proper laboratory examination of the later reactions in a sequence. Initially there are no answers to these supply problems; one just has to run the small-scale reaction and hope that on transfer to larger scale the reaction proceeds as expected.

\section{AAG561, New Process}

The third and final example of process research and development deals with new aspects of nitration chemistry and the sub- sequent reduction of the nitro group to an amine. It also shows an important aspect of process research, the avoidance of toxic intermediates.

The recent multi $100 \mathrm{~kg}$ preparation of the CNS-active substance AAG561 (20) [31] may be seen as an example of how the drawbacks of an early synthesis route, burdened with mutagenic intermediates and purification problems, may bring 'wellknown' heterocyclic chemistry into the focus of interest of process development. A new shortened synthesis route was successfully implemented for AAG561 at an already advanced development stage, which simplified the preparation considerably by replacing the benzthiadiazole $\mathbf{2 1}$ as the cru(Scheme 9).

The established five-step procedure for 21, employing 2-nitro-6-methylaniline (23) as the starting material, was based on S-protection of the phenylendiamine motif for further $\mathrm{Cl} / \mathrm{N}$-functionalisation. However, this involved several mutagenic reagents and intermediates that dictated extra purification operations for the process intermediates and a properly controlled working environment in the pilot plant. Additionally for the last steps towards the active substance $\mathbf{2 0}$ the benzthiadiazole had to be deprotected by reduction and capped with glyoxal in two additional steps. The use of the quinoxaline 22, as a synthon with the full final functionalisation pattern, circumvented all of the inherent problems of the benzthiadiazole route(s).

The straightforward five-step procedure towards the new key intermediate 22 is outlined in Scheme 10 [32].

A priori, the crucial reaction was expected to be the nitration of $\mathbf{2 6}$ to $\mathbf{2 7}$ since the literature precedence with nitration of electron-deficient quinoxalines, or 6-haloquinoxalines, was quite discouraging [32]. cial intermediate with the quinoxaline $\mathbf{2 2}$ 
<smiles>[R]C(O)[C@H](C)CC(=C)[C@@H]1[C@@H](/C=C/C[C@H]2OC(=O)[C@H](C)[C@@H](O)[C@H]2C)[C@H](C)[C@@H](O)[C@@H]1C</smiles>

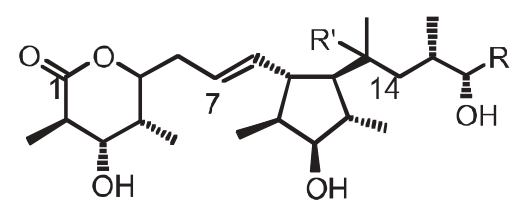

C14 R' = (R)-OH, C14 R' = (S)-OH<smiles>[R]C(O)[C@H](C)C/C(C)=C\[C@H](C)[C@H]1O[C@H](C[C@@H]2OC(=O)[C@H](C)[C@@H](O)[C@H]2C)C=C[C@H]1C</smiles><smiles>C=C/C=C/[C@H](C)[C@H](OC(N)=O)[C@@H](C)[C@H](O)[C@@H](C)C/C(C)=C\[C@@H](C)[C@H](O)[C@H](C)/C=C\[C@H](O)C[C@@H]1OC(=O)[C@H](C)[C@@H](O)[C@H]1C</smiles><smiles>[R]=C=C/C=C\[C@@H](C)[C@H](OC(N)=O)[C@@H](C)[C@H](C)I</smiles>

Fig. 6. Side products from the synthesis of (+)-6

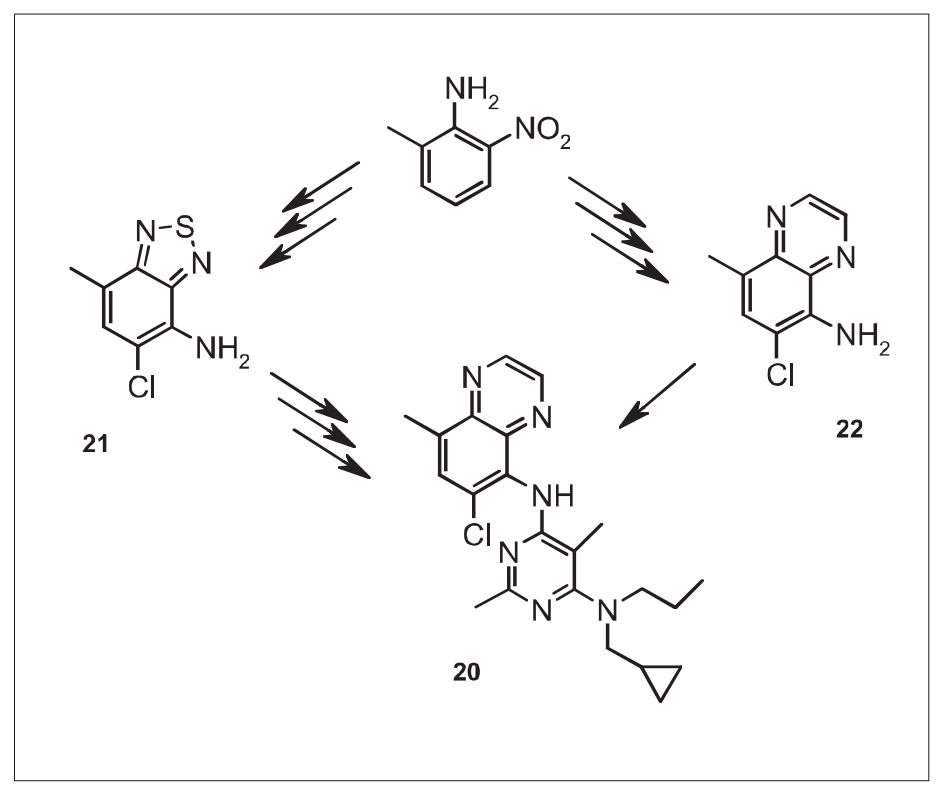<smiles>[R]C(O)[C@H](C)/C=C(\C)[C@@H]1[C@H](/C=C/C[C@H]2OC(=O)[C@H](C)[C@@H](O)[C@H]2C)[C@@H](C)[C@@H](O)[C@@H]1C</smiles><smiles>[R]C(O)[C@H](C)C[C@](C)(O)[C@@H]1[C@H](/C=C/C[C@H]2OC(=O)[C@H](C)[C@@H](O)[C@H]2C)[C@H](C)[C@@H](O)[C@@H]1C</smiles>

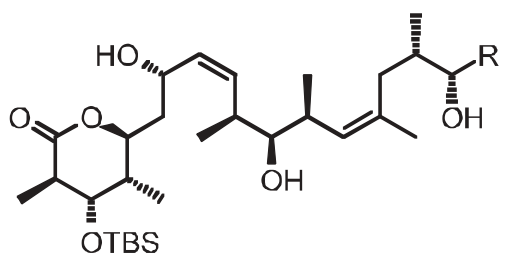

With some delight we found that the 8methylgroup in $\mathbf{2 6}$ (or other 8methylquinoxalines) sufficiently activated the homocyclic ring for a selective and efficient $\mathrm{C}(5)$-nitration in mixed acid at $45^{\circ} \mathrm{C}$. The nitration product 27 could be isolated in high yield and quality by direct crystallization. In the absence of the activating 8methyl group unspecific side reactions dominated. The side-products $(<10 \%)$ in the nitration mixture of $\mathbf{2 6}$, resulting from oxidative dehalogenation at $\mathrm{C}(6) 28$ or chlorination at $\mathrm{C}(7) \mathbf{2 9}$, inferred the presence of nitrylchloride which in turn led to extensive technical measures in order to scrub this volatile and environmentally problematic compound from the process exhaust. Interestingly, the nitration of $\mathbf{2 6}$ in an ionic liquid with nitric acid at $80{ }^{\circ} \mathrm{C}$ takes a completely different turn and leads in a rather sluggish reaction via mononitration at $\mathrm{C}(7) \mathbf{3 0}$ to a $\mathrm{C}(5), \mathrm{C}(7)$-dinitrated product 31, that is not detectable in the mixed acid nitration of $\mathbf{2 6}$ [33].

The manufacture of the quinoxaline 27 was scaled up at the site of an external contractor, according to the new nitration route. For the reduction step $\mathbf{2 7}$ to $\mathbf{2 2}$, as an alternative to our hydrogenation protocol (Raney nickel in ethanol, Scheme 9), a highyielding Bechamp reduction procedure was used for the scale up which unexpectedly led to $2-3 \%$ of the rearranged regioisomer 32 (Scheme 11), presumably due to the extended contact time of the batch with the iron (II) salts formed in the reaction. The regioisomer could not be removed in the work-up, or by an additional recrystallisation, or in the following chemical transformations to the drug substance 20. Finally, an elegant rework procedure was found, which exploited the different solubility of the weak bases $\mathbf{2 2}$ and $\mathbf{3 2}$ in equilibrium with their soluble hydrochloride salts in water/ethanol mixtures, to recrystallise the pure $\mathbf{2 2}$ with an excellent recovery yield [34].

In summary, a simple straightforward process was developed for the key intermediate 22 which is characterised by a good overall yield, a very high purity, readily crystallising intermediates which also contributed to the significantly reduced material flow as compared to the old route.

\section{Conclusions}

Hopefully, with these three examples we have been able to provide a flavour for the breadth and depth of the process chemists' field of expertise. The area is of course much more complex and in process research one must master, or at least be conversant with a wide range of disciplines. 


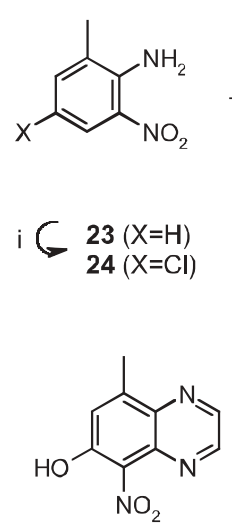

28<smiles>Cc1cc(Cl)cc(N)c1N</smiles><smiles>Cc1cc(Cl)cc2nccnc12</smiles><smiles>[R]c1c(Cl)cc(C)c2nccnc12</smiles>

[25]<smiles>Cc1c(Cl)c(Cl)c([N+](=O)[O-])c2nccnc12</smiles>

29

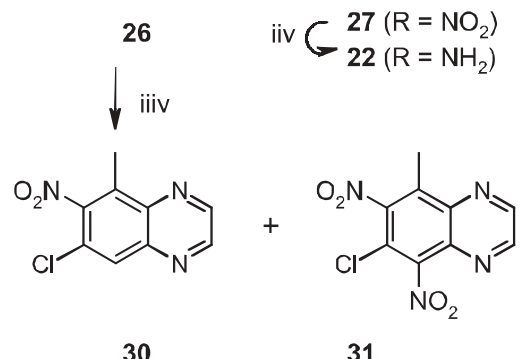

30

[10] E.C. Taylor, R.W. Hendess, J. Am. Chem. Soc. 1964, 86, 951.

[11] A.S. Glickman, US Patent 2'684'976, 1954.

[12] NMR investigations on the synthesis of iminoester $\mathbf{4 b}$ had shown earlier, that at lower temperature, amidine $\mathbf{4 c}$ was the predominant product in the mixture and only by heating above $120^{\circ} \mathrm{C}$ and with excess orthoformate, iminoester $\mathbf{4 b}$ could be obtained.

[13] For a review on Dimroth rearrangements, see e.g. M. Wahren, Z. Chem. 1969, 9(7), 241.

[14] M.A. Zahran, E.B. Pedersen, C. Nielsen, Months. Chem. 1995, 126(11), 1271.

[15] See e.g.: a) D.J. Brown, N.W. Jacobsen, J. Chem. Soc. 1960, 1978; b) E.C. Taylor, P.K. Leaflet, J. Amer. Chem. Soc. 1960, 82, 3147 ; c) Y. Hotcake, Bull. Chem. Soc. Jon. 1970, 43, 3909; d) D.M. Antinovel, L.I. Belen'kii, A.A. Dudinov, M.M. Krayushkin, Khim. Geterotsikl. Soedin 1994, 4, 450.

Scheme 10. Synthesis of the new key intermediate 22



Scheme 11. Regioisomer generating Bechamp-reduction

[1] a) P. Traxler, G. Bold, M. Lang, J. Frei, PCT Int. Appl. WO 9807726, 1998; b) P. Traxler, G. Bold, W. Brill, J. Frei, PCT Int. Appl. WO 9702266, 1997; c) P. Traxler, P. Furet, H. Mett, E. Buchdunger, T. Meyer, N. Lydon, J. Pharm. Belg. 1997, 52(2), 88; d) P. Traxler, Expert Opin. Ther. Pat. 1997, 7(6), 571, and references cited therein; e) R.W. Fischer, M. Misun, Org. Proc. Res. Dev. 2001, 5, 581.

[2] K. Gewald, Z. Chem. 1961, 1, 349.

[3] a) V.I. Shvedov, M.V. Mezentseva, A.N. Grinev, Khim. Geterotsikl. Soedin. 1975, 9, 1217; b) H. Wamhoff, B. Wehling, Synthesis 1976, 51.

[4] H.D. Dakin, R. West, J. Biol. Chem. 1928, 78, 91; H.D. Dakin, R. West, J. Biol. Chem. 1928, 78, 745.

[5] a) G.H. Cleland, C. Niemann, J. Am. Chem. Soc. 1949, 71, 841; b) W. Steglich, G. Höfle, Tetrahedron Lett. 1968, 13, 1619; c) W. Steglich, G. Höfle, Chem. Ber. 1969, 102, 883; d) W. Steglich, G. Höfle, Chem. Ber. 1969, 102, 899; e) W. Steglich, G. Höfle, A.
Prox, Chem. Ber. 1972, 105, 1718; f) R. Knorr, Chem. Ber. 1971, 104, 3633; g) N.L. Allinger, G.L. Wang, B.B. Dewhurst, $J$. Org. Chem. 1974, 39(12), 1730.

[6] This mechanism was originally proposed by Cleland and Niemann (see [5a]); it only applies to the Dakin-West reaction of primary amino acids.

[7] W. Steglich, G. Höfle, Angew. Chem. 1969, 81(23), 1001.

[8] Safety remark: malonodinitrile decomposes autocatalytically at elevated temperatures with an adiabatic temperature rise in excess of $1000{ }^{\circ} \mathrm{C}$ ! The initiation phase of the decomposition is shortened by bases. For these reasons, when performing this type of pyrrole condensation reaction on large scale, malonodinitrile should be the component being added to the sodium hydroxide solution rather than the opposite way to avoid a possible runaway reaction.

[9] Bulk substance was stored in barrels which were flushed with nitrogen to avoid pyrrole oxidation.
[16] M. Misun, R. Fisher, M. Mutz, PCT int. Appl. WO 98/43973, 1998.

[17] a) S.P. Gunasekera, M. Gunasekera, R.E. Longley, G.K. Schulte, J. Org. Chem. 1990, 55, 4912; Correction: S.P. Gunasekera, M. Gunasekera, R.E. Longley, G.K. Schulte, J. Org. Chem. 1991, 56, 1346; b) S.P. Gunasekera, S.A. Pomponi, R.E. Longley, U. S. Patent 5840750, November 24, 1998; c) S.P. Gunasekera, G.K. Paul, R.E. Longley, R.A. Isbrucker, S.A. Pomponi, $J$. Nat. Prod. 2002, 65, 1643.

[18] F.R. Kinder, Jr., K.W. Bair, W. Chen, G. Florence, C. Francavilla, P. Geng, S.P. Gunasekera, Q. Guo, P.T. Lassota, R.E. Longley, M.G. Palermo, I. Paterson, S.A. Pomponi, T.M. Ramsey, L. Rogers, M. Sabio, N. Sereinig, E. Sorensen, R.M. Wang, A. Wright, Abstracts of Papers, $224^{\text {th }}$ American Chemical Society National Meeting, Boston, MA, August 18-22, 2002; MEDI-236.

[19] a) J.B. Nerenberg, D.T. Hung, P.K. Sommers, S.L. Schreiber, J. Amer. Chem. Soc. 1993, 115, 12621; b) D.T Hung, J.B. Nerenber, S.L. Schreiber, J. Amer. Chem. Soc. 1996, 118, 11054; c) A.B. Smith, Y. Qui, D.R. Jones, K. Kobayashi, J. Amer. Chem. Soc. 1995, 117, 12011; d) A.B. Smith, M.D. Kaufmann, T.J. Beauchamp, M.J. LaMarche, H. Arimoto, Org. Lett. 1999, 1, 1823; Additions and corrections: A.B. Smith, M.D. Kaufmann, T. J. Beauchamp, M.J. LaMarche, H. Arimoto, Org. Lett. 2000, 2, 1983; e) A.B. Smith, T.J. Beauchamp, M.J. LaMarche, M.D. Kaufmann, Y. Qui, H. Arimoto, D.R. Jones, K. Kobayashi, J. Amer. Chem. Soc. 2000, 112, 8654; f) S.S. Harried, G. Yang, M.A. Strawn, D.C. Myles, J. Org. Chem. 1997, 62, 6098; g) J.A. Marshall, B.A. Johns, J. Org. Chem. 1998, 63, 7885; h) I. Paterson, G.J. Florence, K. Gerlach, J.P. Scott, Angew. Chem. Int. Ed. 2000, 39, 
377; i) I. Paterson, G.J. Florence, Tetrahedron Lett. 2000, 41, 6935; j) I. Paterson, G.J. Florence, K. Gerlach, J.P. Scott, N. Sereinig, J. Amer. Chem. Soc. 2001, 123, 9535; k) I. Paterson, O. Delgado, G.L. Florence, I Lyothier, J.P. Scott, N. Sereinig, Org. Lett. 2003, 5, 35.

[20] See e.g.: a) C. Francavilla, W. Chen, F.R. Kinder, Jr., Organic Letters 2003, 5, 1233; b) A. Arefolov, J.S. Panek, Org. Lett. 2002, 4, 2397; c) K.A. Shahid, J. Mursheda, M. Okazaki, Y. Shuto, F. Goto, S. Kiyooka, Tetrahedron Lett. 2002, 43, 6377; d) K.A. Shahid, Y.N. Li, M. Okazaki, Y. Shuto, F. Goto, S. Kiyooka, Tetrahedron Lett. 2002, 43, 6373; e) M. Miyazawa, S. Oonuma, K. Maruyama, M. Miyashita, Chem. Lett. 1997, 1191; f) M. Miyazawa, S. Oonuma, K. Maruyama, M. Miyashita, Chem. Lett. 1997, 1193; g) S. BouzBouz, J. Cossy, Org. Lett. 2001, 3, 3995; h) J.M.C. Golec, S. Jones, Tetrahedron Lett. 1993, 34, 8159. i) P.L. Evans, J.M. C. Golec, R.J. Gillespie, Tetrahedron Lett. 1993, 34, 8163; j) J.M.C. Golec, R.J. Gillespie, Tetrahedron Lett. 1993, 34, 8167; k) J.A. Marshall, Z.H. Lu, B.A. Johns, J. Org. Chem. 1998, 63,817 ; 1) G. Yang, D.C. Myles, Tetrahedron Lett. 1994, 35, 1313; m) G. Yang, D.C. Myles, Tetrahedron Lett. 1994, 35, 2503 ; n) I. Paterson, A. Schlapbach, Synlett 1995, 498.

[21] I. Paterson, G.J. Florence, Eur. J. Org. Chem. 2003, 12, 2193.

[22] A.B. Smith, S.B. Freeze, I. Brouard, T. Hirose, Org. Lett. 2003, 5(23), 4405.

[23] a) S.J. Mickel, G.H. Sedelmeier, D. Niederer, R. Daeffler, A. Osmani, K. Schreiner, M. Seeger-Weibel, B. Bérod, K.
Schaer, R. Gamboni, S. Chen, W. Chen, C.T. Jagoe, F.R. Kinder, Jr., M. Loo, K. Prasad, O. Repic, Wen-C. Shieh, Run-M. Wang, L. Waykole, D. Xu, S. Xue, Organic Process Research \& Development 2004, 8(1), 92; b) S.J. Mickel, G.H. Sedelmeier, D. Niederer, F. Schuerch, D. Grimler, G. Koch, R. Daeffler, A. Osmani, A. Hirni, K. Schaer, R. Gamboni, A. Bach, A. Chaudhary, S. Chen, W.C. Chen, B. Hu, C.T. Jagoe, H.Y. Kim, F.R. Kinder, Jr., Y. Liu, Y. Lu, J. McKenna, M. Prashad, T.M. Ramsey, O. Repic, L. Rogers, Wen-C. Shieh, Run-M. Wang, L. Waykole, Organic Process Research \& Development 2004 8(1), 101; c) S.J. Mickel, G.H. Sedelmeier, D. Niederer, F. Schuerch, G. Koch, E. Kuesters, R. Daeffler, A. Osmani, M. Seeger-Weibel, E. Schmid, A. Hirni, K. Schaer, R. Gamboni, A. Bach, S. Chen, W.C. Chen, P. Geng, C.T. Jagoe, H.Y Kim, F.R. Kinder, Jr., G.T. Lee, J. McKenna, T.M. Ramsey, O. Repic, L. Rogers, Wen-C. Shieh, Run-M. Wang, L. Waykole, Organic Process Research \& Development 2004, 8(1), 107; d) S.J. Mickel, G.H. Sedelmeier, D. Niederer, F. Schuerch, M. Seeger-Weibel, K. Schreiner, R. Daeffler, A. Osmani, D. Bixel, O. Loiseleur, J. Cercus, H. Stettler, K. Schaer, R. Gamboni, A. Bach, G.P. Chen, W.C. Chen, P. Geng, G.T. Lee, E. Loesser, J. McKenna, F.R. Kinder, Jr., K. Konigsberger, K. Prasad, T.M. Ramsey, N. Reel, O. Repic, L. Rogers, Wen-C. Shieh, Run-M. Wang, L. Waykole, S. Xue, G. Florence, I. Paterson, Organic Process Research \& Development 2004, 8(1), 113; e) S.J. Mickel, D. Niederer, R. Daeffler, A. Osmani, E.
Kuesters, E. Schmid, K. Schaer, R. Gamboni, W.C. Chen, E. Loesser, F.R. Kinder, Jr., K. Konigsberger, K. Prasad, T.M. Ramsey, O. Repic, Run-M. Wang, G. Florence, I. Lyothier, I. Paterson, Organic Process Research \& Development 2004, 8(1), 122.

[24] J. Chen, K. Zhao, Tetrahedron Lett. 1994, $35,2827$.

[25] H. Arimoto, M.D. Kaufmann, K. Kobayashi, Y. Qiu, A.B. Smith, Synlett. 1998, 765.

[26] Y. Shen, S. Gao, J. Chem. Soc. Perkin Trans. I 1995, 1331.

[27] a) M.H.B. Stowell, J.M. Ueland, R.W. McClard, Tetrahedron Lett. 1990, 31, 3261; b) C. Patois, P. Savignac, Tetrahedron Lett. 1991, 32, 1317; c) S. Hanessian, Y.L. Bennani, D. Delorne, Tetrahedron Lett. 1990, 31, 6461; d) S. Hanessian, Y.L. Bennani, Tetrahedron Lett. 1990, 31, 6465.

[28] C.J. Cowden, I. Paterson, Org. React. 1997, 51, 1 .

[29] D.A. Evans, K.T. Chapman, E.M. Carreira, J. Am. Chem. Soc. 1988, 110, 3560.

[30] S.P. Gunasekera, S.J. Mickel, R. Daeffler, D. Niederer, A.E. Wright, P. Linley, T. Pitts, J. Nat. Prod. 2004, 67(5), xxxx (ASAP).

[31] B.P. Neumann, Patent WO 99/40089, 1999.

[32] W. Marterer, W. Prikoszovich, J. Wiss, M. Prashad, Organic Process Research \& Development 2003, 7, 318.

[33] D. Marchand, B. Wietfeld, W. Marterer, unpublished results.

[34] C. Vogel, W. Marterer, unpublished results. 